\title{
Self-Attentional Credit Assignment for Transfer in Reinforcement Learning
}

\author{
Johan Ferret, Raphaël Marinier, Matthieu Geist and Olivier Pietquin \\ Google Research, Brain Team \\ \{jferret, raphaelm, mfgeist, pietquin\}@ google.com
}

\begin{abstract}
The ability to transfer knowledge to novel environments and tasks is a sensible desiderata for general learning agents. Despite the apparent promises, transfer in RL is still an open and little exploited research area. In this paper, we take a brand-new perspective about transfer: we suggest that the ability to assign credit unveils structural invariants in the tasks that can be transferred to make RL more sample efficient. Our main contribution is SECRET, a novel approach to transfer learning for RL that uses a backward-view credit assignment mechanism based on a self-attentive architecture. Two aspects are key to its generality: it learns to assign credit as a separate offline supervised process and exclusively modifies the reward function. Consequently, it can be supplemented by transfer methods that do not modify the reward function and it can be plugged on top of any RL algorithm.
\end{abstract}

\section{Introduction}

To some, intelligence is measured as the capability of transferring knowledge to unprecedented situations. While the notion of intellect itself is hard to define, the ability to reuse learned information is a desirable trait for learning agents. The coffee test [Goertzel et al., 2012], presented as a way to assess general intelligence, suggests the task of making coffee in a completely unfamiliar kitchen. It requires a combination of advanced features (planning, control and exploration) that would make the task very difficult if not out of scope for the current state-of-the-art Reinforcement Learning (RL) agents to learn. On the other hand, it is solved trivially by humans, who exploit the universally invariant structure of coffee-making: one needs to fetch a mug, find coffee, power the coffee machine, add water and launch the brewing process by pushing the adequate buttons. Thus, to solve the coffee test, transfer learning appears necessary. Were we to possess a random kitchen simulator and a lot of compute, current transfer methods would still fall short of consistently reusing structural information about the task, hence also falling short of efficient adaptation.

Credit assignment, which in RL refers to measuring the individual contribution of actions to future rewards, is by definition about understanding the structure of the task. By structure, we mean the relations between elements of the states, actions and environment rewards. In this work, we investigate what credit assignment can bring to transfer. Encouraged by recent successes in transfer based on supervised methods, we propose to learn to assign credit through a separate supervised problem and transfer credit assignment capabilities to new environments. By doing so, we aim at recycling structural information about the underlying task.

To this end, we introduce SECRET (SElf-attentional CREdit assignment for Transfer), a transferable credit assignment mechanism consisting of a self-attentive sequence-to-sequence model whose role is to reconstruct the sequence of rewards from a trajectory of agent-environment interactions. It assigns credit for future reward proportionally to the magnitude of attention paid to past state-action pairs. SECRET incorporates structural knowledge in the reward function without modifying optimal behavior, as we show in generalization and transfer scenarios that preserve the structure of the task.

Existing backward-view credit assignment methods [Arjona-Medina et al., 2019; Hung et al., 2018] require auxiliary terms to the loss function used to train agents, which can have detrimental effects to the learning process [de Bruin et al., 2018], and rely on an external memory, which hinder the generality of their approach. SECRET does neither. Also, as we show in Sec. 3.1, the architecture we consider for SECRET has interesting properties for credit assignment. We elaborate about our novelty with respect to prior work in Sec. 4. We insist on the fact that the focus of our work is on transfer and that it is not our point to compete on credit assignment capabilities.

We would like to emphasize several aspects about the generality of SECRET: 1) our method does not require any modification to the RL algorithm used to solve the tasks considered, 2) it does not require any modification to the agent architecture either and 3) it does not alter the set of optimal policies we wish to attain. Moreover, our method for credit assignment is offline, and as a result, it can use interaction data collected by any mean (expert demonstrations, replay memories [Lin, 1992], backup agent trajectories...).

Background. We place ourselves in the classical Markov Decision Process (MDP) formalism [Puterman, 1994]. An MDP is a tuple $(S, A, \gamma, R, P)$ where $S$ is a state space, $A$ is an action space, $\gamma$ is a discount factor $(\gamma \in[0,1)), R$ : $S \times A \times S \rightarrow \mathbb{R}$ is a bounded reward function that maps 
state-action pairs to the expected reward for taking such an action in such a state. Note that we include the resulting state in the definition of the reward function over the typical $R: S \times A \rightarrow \mathbb{R}$. This is for consistency with objects defined later on. Finally, $P: S \times A \rightarrow \Delta_{S}$ is a Markovian transition kernel that maps state-action pairs to a probability distribution over resulting states, $\Delta_{S}$ denoting the simplex over $S$.

An RL agent interacts with an MDP at a timestep $t$ by choosing an action $a_{t} \in A$ and receiving a resulting state $s_{t+1} \sim P\left(\cdot \mid s_{t}, a_{t}\right)$ and a reward $r_{t}=R\left(s_{t}, a_{t}, s_{t+1}\right)$. A trajectory $\tau=\left(s_{i}, a_{i}, r_{i}\right)_{i=1, \ldots, T}$ is a set of state-action pairs and resulting rewards accumulated in an episode. A subtrajectory is a portion of trajectory that starts at the beginning of the episode. The performance of an agent is evaluated by its expected discounted cumulative reward $\mathbb{E}\left[\sum_{t=0}^{\infty} \gamma^{t} r_{t}\right]$. In a partially observable MDP (POMDP), the agent receives at each timestep $t$ an observation $o_{t} \sim \mathcal{O}\left(\cdot \mid s_{t}\right)$ that contains partial or noisy information about the underlying state of the environment.

\section{SECRET: Self-Attentional Credit Assignment For Transfer}

SECRET uses previously collected trajectories from environments in a source distribution. A self-attentive sequence model is trained to predict the final reward in subtrajectories from the sequence of observation-action pairs. The distribution of attention weights from correctly predicted nonzero rewards is viewed as credit assignment. In target environments, the model gets applied to a small set of trajectories. We use the credit assigned to build a denser and more informative reward function that reflects the structure of the (PO)MDP. The case where the target distribution is identical to the source distribution (in which we use held-out environments to assess transfer) will be referred to as generalization or in-domain transfer, as opposed to out-of-domain transfer where the source and the target distributions differ.

\subsection{Self-Attentional Credit Assignment}

Credit assignment as offline reward prediction. We learn to assign credit through an offline reward prediction task, based on saved trajectories of agent-environment interactions. We create a sequence-to-sequence (seq2seq) model [Sutskever et al., 2014] that takes as input the sequence of observationaction pairs and has to reconstruct the corresponding sequence of environment rewards. Being offline, the reward prediction task is learned separately from the RL task, and the reward prediction model does not share representations with the agent. This way, the representations learned for credit assignment do not affect or get mixed with the representations learned for control. Operating offline brings several advantages: one can directly interact with the replay memory of agents and even use expert demonstrations or arbitrary saved transitions as a source of supervision, which could be useful in settings where on-policy interactions are costly, such as robotics. We equip our seq2seq model with an attention mechanism [Bahdanau et al., 2015] and view the attention weights of the reward reconstruction task as our primary source of assigned credit. The motivation to do so is that the seq2seq model looks into the past to find predictive signal in order to reconstruct the reward, so observation-action pairs it attends to should be those which reduce its uncertainty about the future, in other words those that explain future reward and should be credited.

On the use of observations. In MDPs, environment states follow the Markov property: they summarize the history of previous interactions and are sufficient to predict the future. As such, predictive models are highly biased towards focusing on the current sequence element, which hinders credit assignment. Under that consideration, when dealing with MDPs, we turn states into observations by applying transformations that hide a certain amount of information from states and break the Markov assumption. For instance, in gridworlds with visual states, we crop the image and get a player-centered image with a given window size. Doing so encourages the model to look into the past to find predictive signal, and allow us to track the relative importance given to each element to reconstruct the credit assigned. In POMDPs, this might be unnecessary depending on the amount of information shared between observations and true states.

Self-attention for credit assignment. Unlike other seq2seq architectures, self-attentive models like Transformers [Vaswani et al., 2017] have direct computational paths between pairs of sequence elements, due to their representations that depend on projections of all sequence elements. This feature is key to long-term credit assignment. As an illustration, consider an RL task where the terminal reward depends only on the first observation, which is drawn randomly. Predicting the reward correctly requires to remember the first observation, which would be very challenging for a recurrent architecture whose memory goes through $O(n)$ transformations, $n$ being the size of the sequence. On the other hand, a self-attentive model directly accesses the value of the initial observation, which makes credit assignment easier.

Reward prediction architecture. We use a Transformer decoder with a single self-attention layer [Lin et al., 2017] and a single attention head. The model input is a sequence of observation-action couples $\left(o_{t}, a_{t}\right)_{t=0, \ldots, T}$. Each observation goes through a series of convolutional layers (for visual inputs) followed by a series of feed-forward layers. Each action representation, a one-hot vector in the discrete action case, is concatenated to the learned observation embedding. Those representations of dimensionality $d_{i}$ are combined with positional encoding (PE), fed to a self-attention layer and then to a position-wise feedforward layer that outputs logits for reward prediction classes. PE encodes the relative positions of sequence elements, see [Vaswani et al., 2017] for details.

Self-attention is an attention mechanism with parameterization $\left(W_{k}, W_{q}, W_{v}\right)$, each matrix belonging to $\mathbb{R}^{d_{i} \times d_{k}}$, that puts sequence elements in relation by computing non-linear similarity scores for all pairs of elements in the sequence. To do so, each sequence element is mapped to a query vector that is matched against keys and values obtained from the previous elements. To be consistent with the goal of assigning credit, the model should not be able to peek into the future. Thus, we restrict the computational window of each sequence element to the information stored in representations of the previous 
elements in the sequence and its own by applying a causal mask $M_{c}$ to the result of the pairwise similarity computations, assigning a value of 0 to masked elements after the softmax.

Let $X=\left(x_{t}\right)_{t=0, \ldots, T} \in \mathbb{R}^{T \times d_{i}}$ denote the input sequence in a matrix form, $x_{t}$ being the result of internal computations of the model on its $t^{\text {th }}$ input. In the same fashion, we note $Z=\left(z_{t}\right)_{t=0, \ldots, T} \in \mathbb{R}^{T \times d_{k}}$ the sequence resulting from the application of self-attention. We then have

$$
Z=\operatorname{softmax}\left(\frac{M_{c} \odot\left(Q K^{T}\right)-C\left(1-M_{c}\right)}{\sqrt{d_{k}}}\right) V,
$$

where $Q=X W_{q} \in \mathbb{R}^{T \times d_{k}}$ stores queries, $K=X W_{k} \in$ $\mathbb{R}^{T \times d_{k}}$ keys, and $V=X W_{v} \in \mathbb{R}^{T \times d_{k}}$ values as linear projections of the input; $d_{k}$ stands for the dimension of the key vectors, $M_{c} \in\{0,1\}^{T \times T}$ is a binary matrix that acts as a causal mask (a lower triangular matrix), $\odot$ is the Hadamard product and $C$ is a large constant $\left(10^{9}\right.$ in practice).

Notably, the resulting observation-action representation can be viewed as a linear combination of the values of previous elements: $z_{t}=\sum_{i=0}^{t} \alpha_{i \leftarrow t} v_{i}$ where $\alpha_{\leftarrow \leftarrow t}=\left(\alpha_{i \leftarrow t}\right)_{i=1, \ldots, t} \propto$ $\exp \left(\left\langle q_{t}, k_{i}\right\rangle / \sqrt{d_{k}}\right)$. The vector $\alpha_{t}$ contains the normalized attention weights for the prediction at timestep $t$ and sums to 1. Since observations contain only a portion of their initial information, the fact that the model succeeds in the prediction task indicates that it reconstructed the missing information from its past. Therefore, attention weights themselves can be viewed as a form of credit assignment, and will be used as such in what follows.

While performing regression on the rewards could also be an option, our experiments found that regression tends to converge to poor local optima. Consequently, we predict the sign of the experienced rewards: $q(r)=\operatorname{sign}(r)$ with $\operatorname{sign}(0)=0$. We chose the sign as the classification target for its invariance to the scale of the rewards. We use a weighted sequential cross-entropy as the loss function over the class-wise model predictions $f_{\theta, c}$, writing $\tau(o, a)$ the subtrajectory of $\tau$ ending with the observation-action couple $(o, a)$ to translate the effect of the binary mask:

$$
\mathcal{L}_{\theta}(\tau)=-\sum_{c \in\{-1,0,1\}} \frac{w(c)}{|\tau|} \sum_{(o, a, r) \in \tau} \mathbb{I}\{q(r)=c\} \log \left(f_{\theta, c}(\tau(o, a))\right) .
$$

We have found class weighting $w(c)$ to be very important in this imbalanced prediction task. It reduces the variance of predictive performance across datasets of sampled trajectories for a given model and hyperparameters.

Generating trajectories. To train SECRET, we generate a dataset of trajectories that contains a certain proportion of successful trajectories. If source environments are simple enough so that the task has sufficient chance to be solved by acting randomly, we use a random policy to generate trajectories. For more complex distributions of environments, we use an RL agent (either trained or in the learning phase) to generate trajectories. We think purely exploratory methods [Ecoffet $e t$ al., 2019] could have advantages over using an RL agent and leave the study of their use for future work.

\subsection{Leveraging Credit Via Reward Shaping}

In this subsection, we explain how we use credit assignment to make learning more sample-efficient.

Reward shaping. In RL, agents often deal with sparse rewards that make the learning process slow. Reward shaping [Ng et al., 1999] is a technique that often aims at densifying the reward so as to improve sample efficiency. It defines a class of reward functions that can be added to the original environment rewards without modifying the set of optimal policies. For a given $\operatorname{MDP} M=(S, A, \gamma, R, P)$, we define a new MDP $M^{\prime}=\left(S, A, \gamma, R^{\prime}, P\right)$ where $R^{\prime}=R+F$ is the shaped reward and $F$ the shaping. The reward shaping theorem states that if there exists a potential function $\phi$ such that $F:\left(s, s^{\prime}\right) \rightarrow \gamma \phi\left(s^{\prime}\right)-\phi(s)$, then $M$ and $M^{\prime}$ admit the same set of optimal policies. With domain knowledge, one can use reward shaping to design more informative reward functions without encouraging unwanted behavior. Nevertheless, shaping rewards requires good priors for the task and the potential function must often be engineered manually.

Since SECRET weighs the contribution of observation-action pairs to future reward, we use it to derive a shaped reward that corresponds to the sum of future reward reachable from the underlying state, weighted by the attention calculated by the model. We explain the process in the following.

Computing the potential function. We define the redistributed return $R_{\tau}^{\leftarrow}$ of a trajectory $\tau$ as:

$$
R_{\tau}^{\leftarrow}(s, a)=\sum_{t=1}^{T} \mathbb{I}\left\{s_{t}=s, a_{t}=a\right\} \sum_{i=t}^{T} \alpha_{t \leftarrow i} r\left(s_{i}, a_{i}\right)
$$

where $\alpha_{i \leftarrow j}$ is the attention weight on $\left(o_{i}, a_{i}\right)$ when predicting the reward $r_{j}$ and $s_{i}$ are environment states. Indeed, SECRET uses observations but we keep the states they are constructed from to compute the potential. In POMDPs, we recover an approximate state from the observation, either manually or through inference. In this work, we use a state constructed manually, see Sec. 3, paragraph Implementation details.

To compute the potential function, we generate a set $D$ of trajectories like described in Sec. 2.1. Since we operate on trajectories, the same state-action pair can appear twice in a sequence and benefit from a different amount of attention, which is why we must include the first summation. In the reward shaping formalism, the potential function $\phi$ depends only on the state. To stay within its bounds, we define $\phi$ as the forwarded redistributed return. It is computed as the following estimate:

$$
\hat{\phi}(s)=\frac{1}{|D|} \sum_{\tau \in D} \sum_{t=1}^{T} \mathbb{I}\left\{s_{t}^{(\tau)}=s\right\} R_{\tau}^{\leftarrow}\left(s_{t-1}^{(\tau)}, a_{t-1}^{(\tau)}\right)
$$

Note that in practice we only redistribute individual rewards that were successfully predicted. Also, some states are generally missing from the data distribution induced by the set of trajectories used. For those states, we set to potential to 0 , which results in a $-\hat{\phi}(s)$ additional reward when transitioning 
to those from the state $s$. As a result, it gives agents incentive to stay on the support of the data distribution unless they encounter high-reward states.

Because it relies on reward shaping, SECRET conserves optimal policies. We empirically find that agents learn faster with the resulting augmented reward function. A way to look at it is that we densify the learning signal and bias the agent towards behaviors that encourage future rewards.

\subsection{Transferring Credit Assignment}

We start by conveying intuition as to why SECRET should transfer to new environments. In fields other than RL, seq2seq models similar to that of SECRET have shown outstanding transfer capabilities [Devlin et al., 2019], even in low-resource settings [Zoph et al., 2016]. In transfer scenarios that preserve the structure of the MDP, the optimal finegrained control sequence can vary drastically from one environment to another. This is why credit assignment is an interesting alternative to the transfer of weights: given an underlying environment state and a specific action, their contribution to future rewards is not fundamentally altered. Such scenarios include specific changes in the state (or observation) distribution and changes to the reward function that preserve the optimal policies. These also include changes in the dynamics of the environment, and though it affects credit assignment, we show later on that SECRET adapts surprisingly well to such scenarios. Another point that motivates the use of our method for transfer is the fact that we keep the representations learned for credit assignment separate from the control representations learned by agents. Indeed, recent work showed that RL representations were not optimal for transfer [de Bruin et al., 2018].

Transfer setting. We argue that transfer should be considered effective when agents learn to solve target tasks efficiently because efficiency gains in the target domain compound while the cost of training in the source is fixed. Hence, we use the Total Target Time Scenario metric [Taylor and Stone, 2009] to assess transfer. Nevertheless, collecting trajectories in the source domain can be costly. We report the number of trajectories used to train SECRET in each scenario.

As before, SECRET is trained on episodes of interaction sampled from the source distribution. In each target environment, we sample multiple trajectories (see the following section for details about the policies used to generate the trajectories). We then compute the attentional potential function by calculating an estimate of the expected redistributed reward, as described in Sec. 2.2.

\section{Experiments}

In this section, we aim to answer the following questions: can SECRET improve the sample efficiency of learning for RL agents? Does it generalize and/or transfer? How does it compare to transfer baselines? Is the credit assigned by SECRET interpretable?

The Triggers environment. We introduce Triggers, an interpretable and customizable environment that we use to assess the quality of the credit inferred with our method. In Triggers, the agent is located in a two-dimensional bounded grid. Its
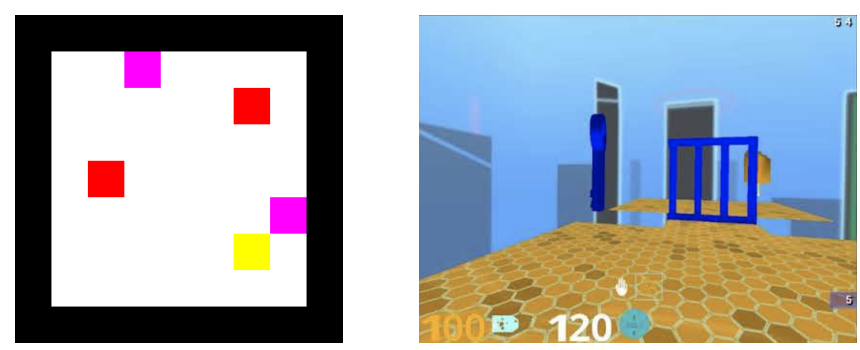

Figure 1: Left: Example of a Triggers environment. Right: Observations from DMLab are first person views.

actions consist solely of moving of one cell in one of the cardinal directions. Any action that would lead the agent outside the boundaries of the environment (as indicated by the walls in the figure) is ignored but still counted as an action taken by the agent. The goal of the agent (represented as a yellow square) is to activate all the switches (red squares) and then collect all the prizes (pink squares). Prizes are the only source of reward and give a -1 penalty unless all switches are activated, in which case they give $a+1$ bonus. Both prizes and switches disappear once collected. The main feature of Triggers is that every positive reward is conditional to the presence of a known subset of states in the agent history, and thus credit assignment can be assessed in a rigorous way. Some instances of Triggers can prove challenging to solve optimally for traditional RL methods since agents have to activate every Triggers before experiencing rewards. Triggers environments being MDPs, we turn their states into observations by cropping the view around the agent. We use $3 \times 3$ windows in all our experiments. Trajectories are generated with random policies.

DMLab keys doors. We use the keys_doors_puzzle 3D environment from DMLab [Beattie et al., 2016] in which the agent must locate keys whose colors indicate the doors they open. It can only possess one key, therefore picking the wrong key prevents it from reaching further rewards. The agent receives as input what would correspond to a first person view of what is in its line of sight. It can move forward, backward and rotate. Each key picked up grants a +1 bonus, equally to each door opened. Independently, a cake rewards the agent by $\mathrm{a}+50$ increase in score when collected. Unlike in Triggers and because the environments are partially observable, we do not apply any transformation to the observations the agent takes as input. In that setup, agents benefit from understanding the link between keys and doors. We hypothesized that SECRET might exploit this relation and reward the agent for picking up keys. To assert this, we modified the setting so that picking up keys does not provide rewards. Additionally, the visual input is richer than the one from Triggers environments and the average number of steps per episode is extended. Finally, agents move and rotate across the room. Since picking up a key does not require to look at the key, it can be hard to know if a key was taken and predict further door opening rewards. Trajectories used to train SECRET are generated with a trained agent.

Implementation details. We use $Q$-learning [Watkins and Dayan, 1992] (tabular, with a learning rate of 0.1 and $\epsilon=0.1$ ) 
for experiments in Triggers except for out-of-domain transfer to environments with modified dynamics where we use DQN [Mnih et al., 2015]. We use PPO [Schulman et al., 2017] for in-domain experiments in DMLab, with identical hyperparameters as in Episodic Curiosity [Savinov et al., 2019], whose code is open-source. Note that we use SECRET with various RL agents, without any change to the algorithm.

For the reward prediction model, we use the same set of hyperparameters in all experiments with little variation. In Triggers experiments, we use 128 units per dense layer, 32 convolutional filters and a single convolutional layer to process partial states. We use a dropout rate of 0.1 after dense layers, a dropout rate of 0.2 in the self-attention mechanism and in the normalization blocks of the Transformer. Class weights in the loss function are set to $w(1)=w(-1)=0.499, w(0)=$ 0.002. In DMLab experiments, we use 16 convolutional filters and two convolutional layers to process partial states, and otherwise identical hyperparameters.

In Triggers, 40000 trajectories are sampled from random policies to train reward prediction models. In DMLab, 10000 trajectories are sampled from a pretrained agent, since random policies cannot be used for transfer: they yield very few if no high-reward trajectories. Still in DMLab, we use a constructed state to compute the potential function in Equation (1) and (2): it is the concatenation of the discretized position and the identifier of the key possessed. Relying on a manually constructed state limits the generality of our approach in POMDPs, but we are confident that this limitation can be addressed by using an estimate of the true state, which we leave for future work.

\subsection{Credit Assignment}

We provide an analysis of the credit inferred by SECRET. The analysis is qualitative and quantitative, since we rely on both visual assessment and binary detection metrics.

The process of evaluating credit assignment in Triggers goes as follows: we first generate trajectories and train the model. We then compare the credit assigned by SECRET on trajectories sampled from held-out environments to a ground truth credit assignment. We build that ground truth by exploiting the exact knowledge of where triggers are. It is a vector that is 0 almost everywhere and 1 on state-action couples that precede the activation of a Triggers. By doing so, we explicitly target the state-action couples whose resulting state is causally linked to the reward experienced later.

We find the redistribution to be near optimal in simple instances of Triggers (see Fig. 2-left): attention concentrates quasi exclusively on state-action pairs that enable the collection of future reward. This is confirmed by precision-recall analysis: we compare binarized attention vectors (values above $\alpha$ are set to 1 ) to ground truth attention vectors (their values are 1 for timesteps that correspond to trigger activations and 0 otherwise). Over the distribution of scenarios considered and for $\alpha=0.2$, it yields an average precision of 0.96 for an average recall of 0.94 .

In keys_doors_puzzle, we adopt the same set of experiments. Since the agent can move backward and spin, in some scenarios it takes a key that is not in its line of sight. In addition, the granularity of the state space is such that off-by-one prediction errors are common but do not hinder
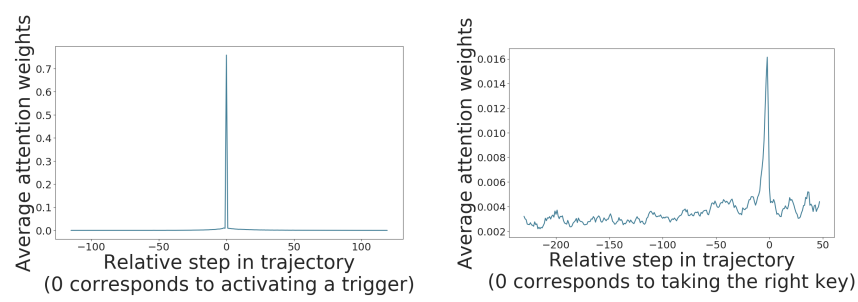

Figure 2: Left: the distribution of attention weights around triggers for correct positive reward predictions in a $8 \times 8$ Triggers maze with 3 triggers and 1 reward. The $\mathrm{x}$-axis denotes the number of steps between the state-action couple receiving attention and the closest moment the agent activated a switch. Right: the distribution of attention weights around keys for correct reward predictions for door traversals in DMLab.
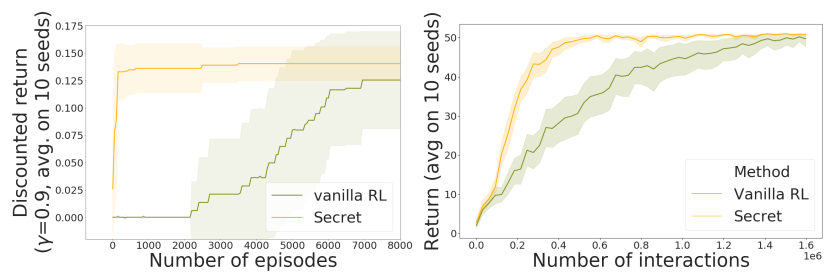

Figure 3: Left: in-domain transfer results on a $8 \times 8$ Triggers with 3 triggers and 1 reward. Right: results in DMLab.

the credit mechanism: attributing credit to the state-action couple preceding the collection of a key or the previous one leads to imperceptible changes in the resulting shaped rewards. Fig. 2-right shows similar results as for Triggers.

\subsection{Transfer}

We then study how we can leverage the inferred credit and transfer representations that are helpful in new scenarios. We show that agents train faster when using shaped rewards from SECRET. As before, the reward model is trained on episodes of interaction in environments sampled from the source distribution. In transfer environments, we sample multiple trajectories, each using the same maze configuration. We then compute the attentional potential function by calculating an estimate of the expected redistributed reward, as described in Sec. 2.2. To evaluate its effect, we compare agents trained from environment rewards to agents that use the resulting shaped reward.

In-domain transfer. For in-domain transfer, we transfer the representations for credit assignment to held-out instances of the same distribution over MDPs. For the Triggers environment, the RL agents are tabular $Q$-learners. For the DMLab environment, we use PPO agents [Schulman et al., 2017] and modify the original task: we do not reward the agent for collecting keys but only to open doors so that the attention can focus on the key positions. Note that it makes the task harder.

As we display in Fig. 3 agents learn visibly faster to solve tasks when benefitting from SECRET in both environments.

Out-of-domain transfer. For out-of-domain transfer we use the Triggers environment and consider two scenarios that are hard for standard agents: transfer to bigger environments and transfer to environments with inverted dynamics (see Fig. 4). 

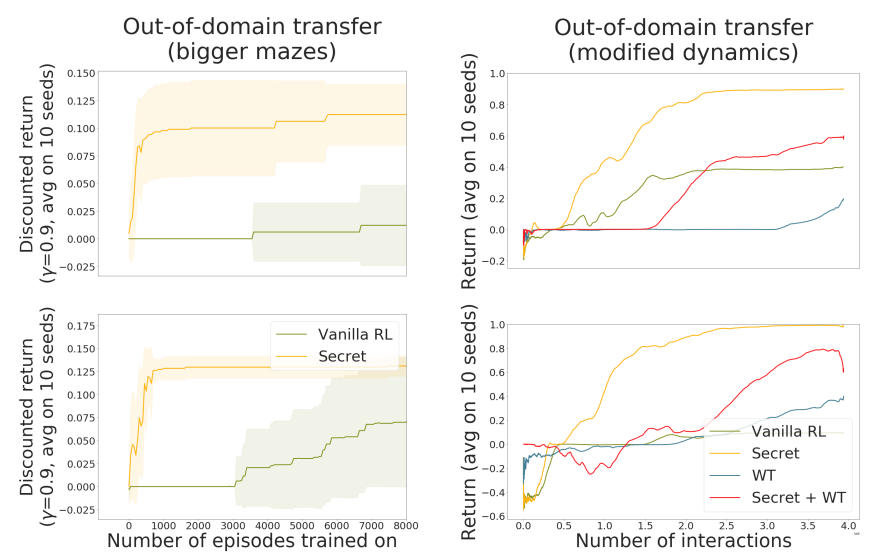

Figure 4: Left: we study transfer to bigger mazes where the structure of the original task is conserved (number of triggers, number of prizes). Environments drawn are $12 \times 12$ grids with 1 trigger and 1 prize for the top figure versus 2 prizes for the bottom one. Environments from the training distribution are $8 \times 8$ grids. Right: the controls of the out-of-domain distribution are inverted (up becomes down, right becomes left). Environments are $8 \times 8$ grids with 1 trigger and 1 prize (top figure) or 2 prizes (bottom figure). The effect of the shaping is exclusively beneficial, while transferring weights from the source task can be detrimental to the learning process.

In the bigger setting, direct weight transfer cannot be used since the visual input has bigger spatial dimensions. On the other hand, SECRET can be used since the transformation we apply to turn states into observations conserves the visual input dimensions. In the inverted dynamics setting, the effect of the agent's actions are inverted, which makes the task hard for transfer methods. In that setting, we compare the transferability of our mechanism to that of the representations learned by an agent equipped with deep function approximation. To this end we use DQN agents and either train them from scratch in the target environments or start from the set of weights learned in the source environments (WT in Fig. 4-right).

In both settings, shaping the rewards assists the agent in learning to solve the task. We display some results in Fig. 4. When transferring to bigger environments, the agent benefits very early on from the shaped reward, while also reaching better asymptotical performance.

\section{Related Work}

Transfer in RL. While a lot of approaches exist in the transfer literature, to the best of our knowledge none explicitly transfer credit assignment capabilities. Previous work aimed at making the training of an agent in the same task more sampleefficient by using a pretrained model as a teacher [Rusu et al., 2016a]. We learn to assign credit as a parallel task that does not modify the representations of the RL agent. Others learn auxiliary reward functions in the hope that they will enable transfer by imposing consistency in the reward [Houthooft et al., 2019]. Although we also learn additional reward signal, it is based on a redistribution of rewards from the environment, which ensures consistency with the original reward function. Transfer is also viewed as learning tasks in a sequential way [Rusu et al., 2016b; Kirkpatrick et al.,
2017] and this suggests to introduce inductive bias to the neural architectures of agents to reduce catastrophic forgetting. Our method does not require to alter the agent's architecture. Other explicitly address the problem of transfer through the lens of multitask learning [Parisotto et al., 2016; Teh et al., 2017] while we stick to learning from an initial distribution of environments. Meta-learning approaches aim to train agents on a distribution of tasks or environments so that their learned skills and representations work across the underlying continuum, and allow for fast adaptation of the agents [Duan et al., 2017; Wang et al., 2016; Finn et al., 2017; Mishra et al., 2018]. In contrast to meta-learning methods, we do not modify the RL algorithm used to train the agent and SECRET is compatible with any core algorithm for RL.

Credit assignment. Previous works investigated the role of attention mechanisms for credit assignment. SAB [Ke et al., 2018] is a sparse attention mechanism used to derive a modified backpropagation algorithm. We draw inspiration from SAB but operate in the RL context without sparsity assumptions about the attention weights. RUDDER [Arjona-Medina et al., 2019] is an online method for credit assignment based on return decomposition. The focus of RUDDER is on online credit assignment while ours is on transfer. Also, our method operates offline and decomposes individual rewards while theirs require a specific exploration scheme, an additional episodic replay buffer, a compute-heavy contribution analysis method and the addition of several auxiliary losses to the objective the RL agent optimizes. In comparison, SECRET is a lightweight method that does not deal with exploration. TVT [Hung et al., 2018] provide an agent with an external memory and the unsupervised task of reconstructing its inputs (both states and rewards). The agent uses memory reads as a way to identify related elements in sequences, and uses those to transfer the value of states providing delayed rewards to the bootstrapping target of contributing elements. In contrast, SECRET makes use of a non-autoregressive architecture, does not reconstruct states, makes use of reward shaping instead of modifying the update function and most importantly does not rely on an external memory. Recall Traces [Goyal et al., 2019] use a generative model that goes backward from high-reward states and samples state-action pairs that could have led to that state. SECRET also works backward from high-reward states but creates links to previous states from existing trajectories instead of sampling them.

\section{Conclusion}

In this work, we investigated the role credit assignment could play in transfer learning and came up with SECRET, a novel transfer learning method that takes advantage of the relational properties of self-attention and transfers credit assignment instead of policy weights. We showed that SECRET led to improved sample efficiency in generalization and transfer scenarios in non-trivial gridworlds and a more complex 3D navigational task. To the best of our knowledge, this is the first line of work in the exciting direction of credit assignment for transfer. We think it would be worth exploring how SECRET could be incorporated into online reinforcement learning methods and leave this for future work. 


\section{References}

[Arjona-Medina et al., 2019] Jose A Arjona-Medina, Michael Gillhofer, Michael Widrich, Thomas Unterthiner, and Sepp Hochreiter. Rudder: Return decomposition for delayed rewards. NeurIPS, 2019.

[Bahdanau et al., 2015] Dzmitry Bahdanau, Kyunghyun Cho, and Yoshua Bengio. Neural machine translation by jointly learning to align and translate. In ICLR, 2015.

[Beattie et al., 2016] Charles Beattie, Joel Z Leibo, Denis Teplyashin, Tom Ward, Marcus Wainwright, Heinrich Küttler, Andrew Lefrancq, Simon Green, Víctor Valdés, Amir Sadik, et al. Deepmind lab. arXiv preprint arXiv:1612.03801, 2016.

[de Bruin et al., 2018] Tim de Bruin, Jens Kober, Karl Tuyls, and Robert Babuška. Integrating state representation learning into deep reinforcement learning. IEEE Robotics and Automation Letters, 2018.

[Devlin et al., 2019] Jacob Devlin, Ming-Wei Chang, Kenton Lee, and Kristina Toutanova. Bert: Pre-training of deep bidirectional transformers for language understanding. NAACL, 2019.

[Duan et al., 2017] Yan Duan, John Schulman, Xi Chen, Peter L. Bartlett, Ilya Sutskever, and Pieter Abbeel. R1\$2\$: Fast reinforcement learning via slow reinforcement learning. ICLR, 2017.

[Ecoffet et al., 2019] Adrien Ecoffet, Joost Huizinga, Joel Lehman, Kenneth O Stanley, and Jeff Clune. Go-explore: a new approach for hard-exploration problems. arXiv preprint arXiv:1901.10995, 2019.

[Finn et al., 2017] Chelsea Finn, Pieter Abbeel, and Sergey Levine. Model-agnostic meta-learning for fast adaptation of deep networks. In $I C M L, 2017$.

[Goertzel et al., 2012] Ben Goertzel, Matt Iklé, and Jared Wigmore. The architecture of human-like general intelligence. In Theoretical Foundations of Artificial General Intelligence. Springer, 2012.

[Goyal et al., 2019] Anirudh Goyal, Philemon Brakel, William Fedus, Timothy Lillicrap, Sergey Levine, Hugo Larochelle, and Yoshua Bengio. Recall traces: Backtracking models for efficient reinforcement learning. ICLR, 2019.

[Houthooft et al., 2019] Rein Houthooft, Yuhua Chen, Phillip Isola, Bradly Stadie, Filip Wolski, OpenAI Jonathan Ho, and Pieter Abbeel. Evolved policy gradients. In NeurIPS, 2019.

[Hung et al., 2018] Chia-Chun Hung, Timothy P. Lillicrap, Josh Abramson, Yan Wu, Mehdi Mirza, Federico Carnevale, Arun Ahuja, and Greg Wayne. Optimizing agent behavior over long time scales by transporting value. CoRR, abs/1810.06721, 2018.

[Ke et al., 2018] Nan Rosemary Ke, Anirudh Goyal, Olexa Bilaniuk, Jonathan Binas, Michael C Mozer, Chris Pal, and Yoshua Bengio. Sparse attentive backtracking: Temporal credit assignment through reminding. In NeurIPS, 2018.

[Kirkpatrick et al., 2017] James Kirkpatrick, Razvan Pascanu, Neil Rabinowitz, Joel Veness, Guillaume Desjardins, Andrei A Rusu, Kieran Milan, John Quan, Tiago Ramalho, Agnieszka GrabskaBarwinska, et al. Overcoming catastrophic forgetting in neural networks. PNAS, 2017.

[Lin et al., 2017] Zhouhan Lin, Minwei Feng, Cícero Nogueira dos Santos, Mo Yu, Bing Xiang, Bowen Zhou, and Yoshua Bengio. A structured self-attentive sentence embedding. In ICLR, 2017.

[Lin, 1992] Long-Ji Lin. Self-improving reactive agents based on reinforcement learning, planning and teaching. Machine learning, 1992.
[Mishra et al., 2018] Nikhil Mishra, Mostafa Rohaninejad, Xi Chen, and Pieter Abbeel. A simple neural attentive meta-learner. In ICLR, 2018.

[Mnih et al., 2015] Volodymyr Mnih, Koray Kavukcuoglu, David Silver, Andrei A Rusu, Joel Veness, Marc G Bellemare, Alex Graves, Martin Riedmiller, Andreas K Fidjeland, Georg Ostrovski, et al. Human-level control through deep reinforcement learning. Nature, 2015.

[Ng et al., 1999] Andrew Y Ng, Daishi Harada, and Stuart Russell. Policy invariance under reward transformations: Theory and application to reward shaping. In ICML, 1999.

[Parisotto et al., 2016] Emilio Parisotto, Jimmy Ba, and Ruslan Salakhutdinov. Actor-mimic: Deep multitask and transfer reinforcement learning. In ICLR, 2016.

[Puterman, 1994] Martin L. Puterman. Markov Decision Processes. Wiley, 1994.

[Rusu et al., 2016a] Andrei A. Rusu, Sergio Gomez Colmenarejo, Çaglar Gülçehre, Guillaume Desjardins, James Kirkpatrick, Razvan Pascanu, Volodymyr Mnih, Koray Kavukcuoglu, and Raia Hadsell. Policy distillation. In ICLR, 2016.

[Rusu et al., 2016b] Andrei A. Rusu, Neil C. Rabinowitz, Guillaume Desjardins, Hubert Soyer, James Kirkpatrick, Koray Kavukcuoglu, Razvan Pascanu, and Raia Hadsell. Progressive neural networks. CoRR, abs/1606.04671, 2016.

[Savinov et al., 2019] Nikolay Savinov, Anton Raichuk, Raphaël Marinier, Damien Vincent, Marc Pollefeys, Timothy Lillicrap, and Sylvain Gelly. Episodic curiosity through reachability. ICLR, 2019.

[Schulman et al., 2017] John Schulman, Filip Wolski, Prafulla Dhariwal, Alec Radford, and Oleg Klimov. Proximal policy optimization algorithms. CoRR, abs/1707.06347, 2017.

[Sutskever et al., 2014] Ilya Sutskever, Oriol Vinyals, and Quoc V Le. Sequence to sequence learning with neural networks. In NeurIPS, 2014.

[Taylor and Stone, 2009] Matthew E Taylor and Peter Stone. Transfer learning for reinforcement learning domains: A survey. JMLR, 2009.

[Teh et al., 2017] Yee Teh, Victor Bapst, Wojciech M Czarnecki, John Quan, James Kirkpatrick, Raia Hadsell, Nicolas Heess, and Razvan Pascanu. Distral: Robust multitask reinforcement learning. In NeurIPS, 2017.

[Vaswani et al., 2017] Ashish Vaswani, Noam Shazeer, Niki Parmar, Jakob Uszkoreit, Llion Jones, Aidan N Gomez, Łukasz Kaiser, and Illia Polosukhin. Attention is all you need. In NeurIPS, 2017.

[Wang et al., 2016] Jane X. Wang, Zeb Kurth-Nelson, Dhruva Tirumala, Hubert Soyer, Joel Z. Leibo, Rémi Munos, Charles Blundell, Dharshan Kumaran, and Matthew Botvinick. Learning to reinforcement learn. CoRR, abs/1611.05763, 2016.

[Watkins and Dayan, 1992] Christopher JCH Watkins and Peter Dayan. Q-learning. Machine learning, 1992.

[Zoph et al., 2016] Barret Zoph, Deniz Yuret, Jonathan May, and Kevin Knight. Transfer learning for low-resource neural machine translation. ACL 2016, 2016. 